\title{
Papilledema in children with hydrocephalus: incidence and associated factors
}

\author{
Haeng Jin Lee, MD, ${ }^{1}$ Ji Hoon Phi, MD, PhD, ${ }^{2}$ Seung-Ki Kim, MD, PhD, ${ }^{2}$ Kyu-Chang Wang, MD, PhD, ${ }^{2}$ \\ and Seong-Joon Kim, MD, PhD'
}

\begin{abstract}
2Division of Pediatric Neurosurgery, Seoul National University Children's Hospital; and 'Department of Ophthalmology, Seoul National University College of Medicine, Seoul, Republic of Korea
\end{abstract}

\begin{abstract}
OBJECTIVE The aim of this study was to report the incidence of and the factors associated with papilledema in children with hydrocephalus.

METHODS Patients younger than 15 years of age who had been diagnosed with hydrocephalus and treated by extraventricular drainage or ventriculoperitoneal shunt surgery between 2005 and 2015 were retrospectively reviewed. Factors including patient age and sex, etiology of hydrocephalus, duration of signs or symptoms, intracranial pressure (ICP), and presence of papilledema were evaluated.
\end{abstract}

RESULTS Forty-six patients, whose mean age was $6.3 \pm 4.7$ years, were included in the study. The 19 patients without papilledema had a mean age of $2.7 \pm 2.7$ years, and the 27 patients with papilledema had a mean age of $8.8 \pm 4.2$ years ( $p<0.001)$. The mean ICP was $19.9 \pm 10.0 \mathrm{~cm} \mathrm{H}_{2} \mathrm{O}$ among those without papilledema and $33.3 \pm 9.1 \mathrm{~cm} \mathrm{H}_{2} \mathrm{O}$ among those with papilledema $(p<0.001)$. The mean duration of signs or symptoms was $3.0 \pm 4.6$ months in the patients without papilledema and $3.4 \pm 3.9$ months in those with papilledema $(p=0.704)$. The patients with papilledema were older and presented with higher ICP than those without. The causes of hydrocephalus were tumor (59\%), congenital anomaly (19\%), hemorrhage (13\%), and infection (9\%).

CONCLUSIONS Papilledema was more common in patients who were older, who had higher ICP, and whose hydrocephalus had been induced by brain tumor. However, since papilledema was absent in $41 \%$ of the children with hydrocephalus, papilledema's absence does not ensure the absence of hydrocephalus, especially in younger patients.

https://thejns.org/doi/abs/10.3171/2017.2.PEDS16561

KEY WORDS papilledema; disc swelling; hydrocephalus

$\mathrm{H}$ YDROCEPHALUS is caused by an imbalance between the production and absorption of cerebrospinal fluid; it can cause severe morbidity and death in children. Systemic symptoms and signs such as headache, nausea, vomiting, irritability, fatigue, and increased head circumference are useful in diagnosing hydrocephalus; however, they are not sensitive or specific indicators. ${ }^{19}$ These symptoms can be absent, particularly if intracranial pressure (ICP) rises slowly. Furthermore, brain imaging can often be an unreliable guide for detecting hydrocephalus..$^{13}$ Papilledema is considered to be one of the most important signs of hydrocephalus and a critical determinant of the management strategy. $4,5,9,13,15$

Previous reports have described the clinical implications of papilledema in adults with ventriculomegaly or in children with shunt failure. ${ }^{13,14}$ The incidence of papilledema was $14 \%-50 \%$ in children with shunt failure ${ }^{5,14}$ and approximately $65 \%$ in children with primary brain tumors. ${ }^{1}$ However, there are few reports on papilledema in children with hydrocephalus or their characteristics just prior to the first procedure such as shunt surgery. In the present study, we investigated the incidence of papilledema and the factors associated with papilledema in children with hydrocephalus.

\section{Methods}

The institutional review board (IRB) approved this study, and the study protocol followed the tenets of the Declaration of Helsinki. The IRB granted a waiver of 
consent for the study. We performed a retrospective chart review of patients younger than 15 years of age who had been diagnosed with hydrocephalus and treated via extraventricular drainage or ventriculoperitoneal shunt insertion, between 2005 and 2015 at our hospital. A neurosurgeon had determined the diagnosis on the basis of physical findings such as increased head circumference or imaging findings such as enlarged ventricles on brain ultrasonography, CT, or MRI. We evaluated factors including age at diagnosis, sex, etiology of hydrocephalus, duration of signs or symptoms, ICP, and the presence of papilledema. We excluded patients with a history of ophthalmic surgery or of a congenital or acquired ophthalmic condition such as optic nerve disease, glaucoma, media opacity, or cataract.

A radiologist reviews the neuroimaging data, and a neurosurgeon measures ICP during procedures such as extraventricular drainage or ventriculoperitoneal shunt surgery. The degree of ventriculomegaly is quantified using a frontooccipital horn ratio..$^{10}$ To estimate ICP, the opening pressure at the ventricular puncture is measured, though it may be a little higher than in the awake state with spontaneous respiration. The patient is placed supine under general anesthesia with endotracheal intubation and normal-range arterial $\mathrm{CO}_{2}$ level $(38-42 \mathrm{~mm} \mathrm{Hg})$. After ventricular puncture at any site, while minimizing cerebrospinal fluid leakage, the ventricular catheter is connected to an approximately $50-\mathrm{cm}$-long prefilled saline column whose upper end is open. When the column is held upright, the fluid level gradually goes down if the ICP is not exceptionally high. After confirming stabilization and oscillation of the fluid level in the saline column, the height of the fluid level is measured from the presumed foramen of Monro.

Fundus examination is performed just prior to the neurosurgical procedure. Using direct or indirect ophthalmoscopy, an experienced ophthalmologist assesses the optic disc for the presence of swelling; the optic disc is graded as normal if it appears flat with a discrete margin. Papilledema is considered to be present if there is disc elevation, retinal vessel obscuration, or blurred disc margin. If photographs (RetCam, MLI Inc.) are obtained, the disc is graded according to the modified Frisén Scale. The scale characterizes disc swelling as Grades $0-5$, representing increasing severity of optic disc edema: Grade 0 , normal optic disc; Grade 1, minimal degree of edema with C-shaped halo of opacification of the optic disc border; Grade 2, low degree of edema such as circumferential halo of the optic disc border; Grade 3, moderate degree of edema obscuring at least 1 segment of a major vessel leaving the optic disc; Grade 4, marked degree of edema obscuring at least 1 segment of a major vessel on the optic disc; and Grade 5 , severe degree of edema with total obscuration of vessels on and leaving the optic disc. ${ }^{16}$

Patients were divided into 2 groups depending on the presence or absence of papilledema and were subsequently analyzed for factors associated with papilledema. The independent t-test, Pearson chi-square test, Fisher exact test, Kruskal-Wallis test, logistic regression analysis, and linear regression analysis were used for statistical analyses with IBM SPSS software (version 12.0, IBM SPSS Inc.), and $\mathrm{p}$ values $<0.05$ were considered statistically significant.

\section{Results}

\section{Characteristics of Patients}

Four hundred nineteen patients younger than 15 years were diagnosed with hydrocephalus and treated by extraventricular drainage or ventriculoperitoneal shunt insertion in the neurosurgery department between 2005 and 2015. Children who did not have a fundus examination or who lacked ICP records were excluded, leaving 46 patients eligible for inclusion in our study. The mean age was 6.3 \pm 4.7 years, and 26 patients were male. In 10 patients, the ophthalmological examination showed the first indication of raised ICP; in the remaining patients, ophthalmological examination was requested after neuroimaging had shown a brain lesion such as a tumor. Additionally, every patient presented with enlarged ventricles on brain imaging; the mean frontooccipital horn ratio was $0.5 \pm 0.1$.

The group with papilledema included 27 patients (59\%) with a mean age of $8.8 \pm 4.2$ years. The group without papilledema consisted of 19 patients $(41 \%)$ with a mean age of $2.7 \pm 2.7$ years $(\mathrm{p}<0.001)$. The mean ICP was $19.9 \pm$ $10.0 \mathrm{~cm} \mathrm{H}_{2} \mathrm{O}$ in the patients without papilledema and 33.3 $\pm 9.1 \mathrm{~cm} \mathrm{H}_{2} \mathrm{O}$ in those with papilledema $(\mathrm{p}<0.001)$. The mean duration of signs or symptoms was $3.0 \pm 4.6$ months in patients without papilledema and $3.4 \pm 3.9$ months in those with papilledema $(\mathrm{p}=0.704$; Table 1$)$.

\section{Causes of Hydrocephalus}

The patients were further subdivided according to the cause of hydrocephalus; 27 patients presented with brain tumor, 9 with congenital anomaly, 6 with brain hemorrhage, and 4 with brain infection. There were no differences in the duration of signs or symptoms among the 4 groups $(p=0.169)$; however, patients with hydrocephalus caused by brain tumor were older and presented with higher ICP than patients in the other hydrocephalus groups. Additionally, a higher incidence of papilledema was observed in patients with hydrocephalus due to brain tumor than in the other groups: $81 \%$ of patients with brain tumor, $33 \%$ of patients with congenital anomaly, $17 \%$ of patients with brain hemorrhage, and $25 \%$ of patients with brain infection ( $\mathrm{p}<0.001$; Table 2$)$.

In the group presenting with brain tumor, there were 12 patients with medulloblastoma, 6 with pilocytic astrocytoma, 3 with germinoma, 3 with mixed germ cell tumor, 2 with ependymoma, and 1 with glioblastoma. Moreover, $70 \%$ of the brain tumors were located infratentorially, $15 \%$ supratentorially, and $15 \%$ in both locations. The specific types, sizes, and locations of the brain tumors were not significantly associated with the presence of papilledema.

In the group presenting with brain hemorrhage, the causes of hemorrhage were nontraumatic subdural hemorrhage, or intraventricular hemorrhage in preterm infants.

\section{Factors Associated With the Presence and Severity of Papilledema}

Patient age, sex, ICP, and duration of signs or symptoms were analyzed using logistic regression analysis. An increased age and increased ICP were significantly associated with the presence of papilledema (Table 3); therefore, we categorized patients by age and ICP. With an age more 
TABLE 1. Summary of characteristics in patients with hydrocephalus

\begin{tabular}{|c|c|c|c|c|}
\hline Parameter & Total & No Papilledema & Papilledema & p Value \\
\hline No. of patients & 46 & $19(41 \%)$ & $27(59 \%)$ & \\
\hline Mean age in yrs (range) & $6.3 \pm 4.7(0.1-15.0)$ & $2.7 \pm 2.7(0.1-9.0)$ & $8.8 \pm 4.2(2.0-15.0)$ & $<0.001^{*}$ \\
\hline $\operatorname{Sex}(M: F)$ & $26: 20$ & $9: 10$ & $17: 10$ & $0.371 \dagger$ \\
\hline Mean ICP in $\mathrm{cm} \mathrm{H}_{2} \mathrm{O}$ (range) & $27.8 \pm 11.5(4.0-48.0)$ & $19.9 \pm 10.0(4.0-40.0)$ & $33.3 \pm 9.1(16.0-48.0)$ & $<0.001^{*}$ \\
\hline Mean duration of signs or symptoms in mos (range) & $3.2 \pm 4.2(0.1-20.0)$ & $3.0 \pm 4.6(0.1-20.0)$ & $3.4 \pm 3.9(0.5-13.0)$ & $0.704^{*}$ \\
\hline Mean frontooccipital horn ratio & $0.5 \pm 0.1(0.4-0.7)$ & $0.5 \pm 0.1(0.4-0.7)$ & $0.5 \pm 0.1(0.4-0.7)$ & $0.352^{*}$ \\
\hline Causes of hydrocephalus & & & & $<0.001 \ddagger$ \\
\hline Brain tumor & $27(59 \%)$ & 5 & 22 & \\
\hline Congenital anomaly & $9(19 \%)$ & 6 & 3 & \\
\hline Brain hemorrhage & $6(13 \%)$ & 5 & 1 & \\
\hline Brain infection & $4(9 \%)$ & 3 & 1 & \\
\hline
\end{tabular}

Continuous variables are reported as the mean \pm standard deviation.

* $p$ value calculated from independent t-test.

$\dagger p$ value calculated from Pearson chi-square test.

$\ddagger \mathrm{p}$ value calculated from Fisher exact test.

than 2 years old, papilledema became more likely $(\mathrm{p}<$ 0.001; Fig. 1). The distribution of ICP revealed that papilledema was more common with higher ICP $(p=0.001$; Fig. 2).

Among the 27 patients with papilledema, there were 16 patients whose fundus photographs had been obtained using the RetCam. On evaluation of these images, disc swelling was graded according to the modified Frisén Scale. Six patients had Grade 1 edema, 5 had Grade 2, 3 had Grade 3, and 2 had Grade 4. There were no significant factors associated with the severity of papilledema.

\section{Discussion}

Papilledema is one of the most important signs of hydrocephalus; if undetected, it can lead to irreversible damage such as optic atrophy. However, the absence of papilledema could be a false-negative sign; in our study, papilledema was absent in $41 \%$ of the children with hydrocephalus. Allen et al. ${ }^{1}$ also reported that papilledema was absent in $35 \%$ of children with hydrocephalus due to primary brain tumor. These authors asserted that measuring the head circumference was essential for these younger children.
Our findings showed that children without papilledema were younger than those with papilledema. The absence of papilledema in younger children with hydrocephalus may be attributable to the presence of open fontanelles. In this study, 9 patients had an open fontanelle at the time of the procedure and they all presented with a normal optic disc. Because the fontanelle is open, the infant's skull can expand and provide protection against the increased ICP, so that the rise in ICP is insufficient to cause papilledema. Indeed, this is the known explanation for the low incidence of papilledema among children with congenital hydrocephalus..$^{1,6,14}$ In addition, Hayreh ${ }^{7,8}$ stated that optic disc edema occurs mechanically when ICP rises within the confined skull cavity. Authors of other studies have also reported that the absence of papilledema provided no definitive information about ICP, so clinicians should be aware that a flat optic disc does not preclude the possibility of increased ICP. $3,5,14$

Nonetheless, the presence of papilledema is one of the most useful indicators for a preoperative diagnosis of raised ICP. If papilledema is present in children with signs and symptoms such as nausea, vomiting, and headache, they should be referred for further neurological examina-

TABLE 2. Comparison of groups subdivided according to causes of hydrocephalus

\begin{tabular}{|c|c|c|c|c|c|}
\hline Parameter & Tumor & Congenital Anomaly & Hemorrhage & Infection & p Value \\
\hline No. of patients & 27 & 9 & 6 & 4 & \\
\hline Mean age in yrs (range) & $9.1 \pm 3.8(2.0-15.0)$ & $1.7 \pm 1.3(0.3-3.0)$ & $3.1 \pm 3.5(0.1-10.0)$ & $2.0 \pm 1.2(1.0-3.0)$ & $<0.001^{*}$ \\
\hline $\operatorname{Sex}(M: F)$ & $19: 8$ & $3: 6$ & $3: 3$ & $1: 3$ & $0.121 \dagger$ \\
\hline Mean ICP in $\mathrm{cm} \mathrm{H}_{2} \mathrm{O}$ (range) & $32.4 \pm 10.0(15.0-48.0)$ & $24.1 \pm 9.1(10.0-36.0)$ & $20.7 \pm 12.7(5.0-40.0)$ & $15.5 \pm 9.8(4.0-28.0)$ & $0.004^{*}$ \\
\hline $\begin{array}{l}\text { Mean duration of signs or symptoms in } \\
\text { mos (range) }\end{array}$ & $2.6 \pm 3.5(0.5-13.0)$ & $5.3 \pm 3.4(0.2-10.0)$ & $1.6 \pm 1.4(0.2-4.0)$ & $5.7 \pm 9.6(0.1-20.0)$ & $0.169^{*}$ \\
\hline Mean frontooccipital horn ratio (range) & $0.5 \pm 0.7(0.4-0.6)$ & $0.6 \pm 0.1(0.4-0.7)$ & $0.5 \pm 0.1(0.4-0.7)$ & $0.5 \pm 0.1(0.4-0.7)$ & $0.169^{*}$ \\
\hline Presence of papilledema & $22(81 \%)$ & $3(33 \%)$ & $1(17 \%)$ & $1(25 \%)$ & $<0.001 \dagger$ \\
\hline
\end{tabular}

* $p$ value calculated from Kruskal-Wallis test.

$\dagger p$ value calculated from Fisher exact test. 
TABLE 3. Factors associated with the presence of papilledema, analyzed by logistic regression analysis

\begin{tabular}{lccc}
\hline \multicolumn{1}{c}{ Factor } & $\operatorname{Exp}(\mathrm{B})$ & $\mathrm{p}$ Value & $95 \% \mathrm{Cl}$ \\
\hline Increased age & 1.584 & 0.017 & $1.086-2.311$ \\
\hline Sex & 0.571 & 0.592 & $0.074-4.430$ \\
\hline Increased ICP & 1.112 & 0.048 & $1.001-1.235$ \\
\hline $\begin{array}{c}\text { Increased duration of } \\
\text { signs or symptoms }\end{array}$ & 1.211 & 0.146 & $0.935-1.569$ \\
\hline
\end{tabular}

tion or neuroradiological evaluation. ${ }^{9}$ Additionally, younger patients with posterior fossa tumors have a higher risk for the development of persistent hydrocephalus. ${ }^{12}$ Therefore, frequent and continual follow-up examination is particularly important for children.

Primary brain tumors are the second most common solid tumors in children. In previous studies, the location of the primary brain tumor has been noted to vary with age: $70 \%$ were classified as supratentorial in adults, whereas $70 \%$ were classified as infratentorial in children., ${ }^{2,12}$ As the tumor enlarges within the confined skull cavity, the ventricular system, which is responsible for drainage of cerebrospinal fluid, can become obstructed. This problem is especially common with brainstem and cerebellar tumors, which block the third ventricle, the aqueduct of Sylvius, or the fourth ventricle, resulting in obstructive hydrocephalus. In the present study, the 27 patients with hydrocephalus caused by brain tumor presented with a higher prevalence of papilledema when compared with the other hydrocephalus groups. Furthermore, $86 \%$ of patients with a brain tumor involved the brainstem or cerebellum. This would help to account for the higher prevalence of papilledema evident in this group as compared with other groups.

Previous studies have reported that the development of papilledema requires a critical duration of ICP elevation..$^{11,14,17,18}$ Nazir et al..$^{14}$ stated that the patients with papilledema had a longer duration of symptoms than those with a flat optic disc, supporting the assertion that the duration of ICP elevation is an important factor in the devel-

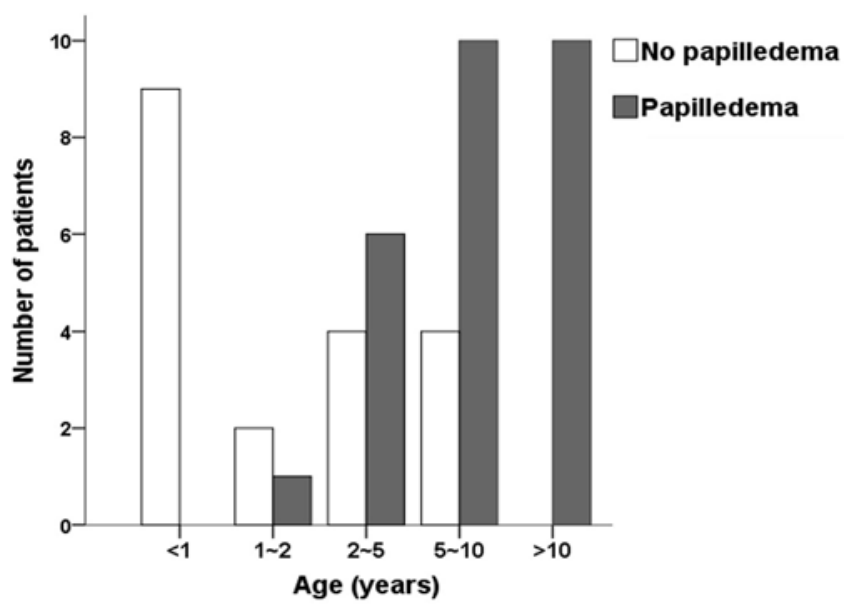

FIG. 1. Bar graph showing patients categorized by age and the presence of papilledema. When age was more than 2 years, the papilledema became more likely $(p<0.001)$.

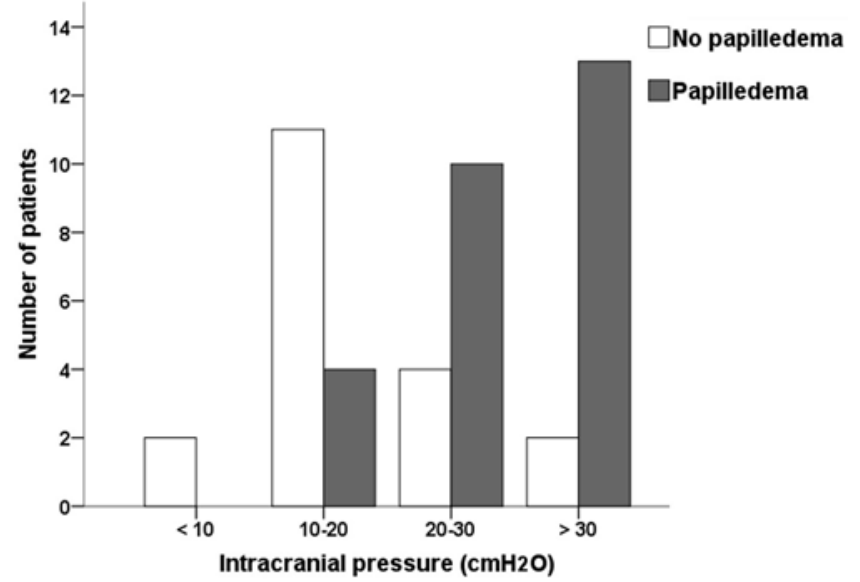

FIG. 2. Bar graph showing patients categorized by ICP and the presence of papilledema. The distribution of ICP revealed that papilledema was more common with higher ICP $(p=0.001)$.

opment of papilledema. Moreover, there have been other reports about the low incidence of papilledema in patients with acute elevations in ICP.,11,17,18 The primary change associated with papilledema is axonal swelling caused by raised ICP resulting in impairment of the axoplasmic flow. If there were an acute elevation in ICP, there would be a blockage of both the anterograde and the retrograde axoplasmic flow. This may not result in disc swelling, suggesting that papilledema is not a sensitive sign of acute ICP elevation.

In this study, among the patients without papilledema, the factors related to brain hemorrhage and infection were more common than other factors. Even though there was no significant difference in the mean duration of signs or symptoms between the 2 groups with and without papilledema, the duration of brain hemorrhage and infection were relatively shorter than that of brain tumors or congenital anomalies. This difference in duration may account for the difference in the incidence of papilledema. However, to have a more definitive account for the differences in duration, further studies including a larger patient cohort would be needed.

This study has some limitations. First, it is a retrospective study, and we included only those patients whose fundus examination had been performed just prior to the neurosurgical procedure; patients without the fundus examination were excluded. The absence of such an examination was attributable to either insufficient time or circumstances that did not allow for examination prior to the shunt procedure (such as in cases of emergency). Additionally, we excluded patients whose ICP at the time of the neurosurgical procedure was undocumented. Because of the small number of patients, there may be selection bias. Second, as the presence of papilledema was assessed subjectively, it is possible that we missed some cases of mild papilledema or misdiagnosed pseudopapilledema as true papilledema. We did not follow up the fundus examination to distinguish between true and pseudopapilledema after the shunting procedure. In addition, there could be interobserver reliability that we should consider. However, brain imaging for every patient showed en- 
larged ventricles, and we tried to make a precise diagnosis depending on the fundus examination performed by an experienced ophthalmologist. Additional investigations including a larger number of patients are needed to explain all aspects of papilledema.

\section{Conclusions}

In summary, patients who were older, who had higher ICP, and whose hydrocephalus was induced by brain tumor presented with a higher incidence of papilledema. The presence of papilledema must be interpreted as a definitive sign of increased ICP. However, for younger patients with hydrocephalus, the absence of papilledema does not exclude hydrocephalus. To make earlier diagnoses of hydrocephalus, clinicians cannot rely on a single finding. They must consider the patient's symptoms, systemic signs, and ocular signs as well as neuroimaging findings to control ICP and prevent loss of vision in a timely fashion.

\section{References}

1. Allen ED, Byrd SE, Darling CF, Tomita T, Wilczynski MA: The clinical and radiological evaluation of primary brain tumors in children, Part I: Clinical evaluation. J Natl Med Assoc 85:445-451, 1993

2. Asai A, Hoffman HJ, Hendrick EB, Humphreys RP, Becker LE: Primary intracranial neoplasms in the first year of life. Childs Nerv Syst 5:230-233, 1989

3. Chou SY, Digre KB: Neuro-ophthalmic complications of raised intracranial pressure, hydrocephalus, and shunt malfunction. Neurosurg Clin N Am 10:587-608, 1999

4. Corbett JJ: Neuro-ophthalmologic complications of hydrocephalus and shunting procedures. Semin Neurol 6:111-123, 1986

5. Gaston H: Ophthalmic complications of spina bifida and hydrocephalus. Eye (Lond) 5:279-290, 1991

6. Ghose S: Optic nerve changes in hydrocephalus. Trans Ophthalmol Soc U K 103:217-220, 1983

7. Hayreh SS: Pathogenesis of optic disc edema in raised intracranial pressure. Prog Retin Eye Res 50:108-144, 2016

8. Hayreh SS: Pathogenesis of optic disc oedema in raised intracranial pressure. Trans Ophthalmol Soc U K 96:404407, 1976

9. Katz DM, Trobe JD, Muraszko KM, Dauser RC: Shunt failure without ventriculomegaly proclaimed by ophthalmic findings. J Neurosurg 81:721-725, 1994

10. Kulkarni AV, Drake JM, Armstrong DC, Dirks PB: Measurement of ventricular size: reliability of the frontal and occipital horn ratio compared to subjective assessment. Pediatr Neurosurg 31:65-70, 1999
11. Levatin P, Raskind R: Delayed appearance of papilledema. Can J Ophthalmol 8:451-455, 1973

12. Lin CT, Riva-Cambrin JK: Management of posterior fossa tumors and hydrocephalus in children: a review. Childs Nerv Syst 31:1781-1789, 2015

13. Mizrachi IB, Trobe JD, Gebarski SS, Garton HJ: Papilledema in the assessment of ventriculomegaly. J Neuroophthalmol 26:260-263, 2006

14. Nazir S, O’Brien M, Qureshi NH, Slape L, Green TJ, Phillips PH: Sensitivity of papilledema as a sign of shunt failure in children. J AAPPOS 13:63-66, 2009

15. Newman NJ: Bilateral visual loss and disc edema in a 15-year-old girl. Surv Ophthalmol 38:365-370, 1994

16. Scott CJ, Kardon RH, Lee AG, Frisén L, Wall M: Diagnosis and grading of papilledema in patients with raised intracranial pressure using optical coherence tomography vs clinical expert assessment using a clinical staging scale. Arch Ophthalmol 128:705-711, 2010

17. Selhorst JB, Gudeman SK, Butterworth JF IV, Harbison JW, Miller JD, Becker DP: Papilledema after acute head injury. Neurosurgery 16:357-363, 1985

18. Steffen H, Eifert B, Aschoff A, Kolling GH, Völcker HE: The diagnostic value of optic disc evaluation in acute elevated intracranial pressure. Ophthalmology 103:1229-1232, 1996

19. Watkins L, Hayward R, Andar U, Harkness W: The diagnosis of blocked cerebrospinal fluid shunts: a prospective study of referral to a paediatric neurosurgical unit. Childs Nerv Syst 10:87-90, 1994

\section{Disclosures}

The authors report no conflict of interest concerning the materials or methods used in this study or the findings specified in this paper.

\section{Author Contributions}

Conception and design: SJ Kim, Phi, SK Kim, Wang. Acquisition of data: all authors. Analysis and interpretation of data: all authors. Drafting the article: Lee. Critically revising the article: SJ Kim. Reviewed submitted version of manuscript: SJ Kim, Lee. Approved the final version of the manuscript on behalf of all authors: SJ Kim. Statistical analysis: Lee. Study supervision: SJ Kim.

\section{Correspondence}

Seong-Joon Kim, Department of Ophthalmology, Seoul National University Hospital, 101 Daehak-Ro, Jongno-Gu, Seoul 110-744, Republic of Korea. email: ophjun@snu.ac.kr. 